\title{
Nonlinear Rheology in Glassy Polymers
}

\author{
Yukuo NANZAI \\ Department of Mechanical and Physical Engineering, \\ Graduate School of Osaka City University, Sumiyoshi-ku, Osaka 558-8585, Japan \\ (Received : October 12, 2005)
}

\begin{abstract}
Application of a rate analysis to post-yield plastic flow in glassy polymers has been carried out through a novel approach. The rate analysis verified that during the yield process the structure of fully annealed glass initially in quasiequilibrium was isothermally changed into various non-equilibrium melt-like structures depending on deformation rates and temperatures. It was shown that this was the case also for the polymer glass at temperatures far below the glass transition point. Structural relaxation in such mechanically induced non-equilibrium structures was illustrated using data of time-dependent mechanical property, differential scanning calorimetry and thermally stimulated deformation recovery. It was found that the strain energy given to glassy polymers during their yielding process was locked in tightly due to structural relaxation of the non-equilibrium structure. Applicability of the non-equilibrium structure to cold working of glassy polymers was discussed.
\end{abstract}

Key Words: Glassy polymer / Plastic flow / Rate Analysis / Isothermal structural transition / Aging / Cold working

\section{INTRODUCTION}

Plastic deformation in glassy polymers is a typical nonlinear phenomenon as demonstrated by many workers ${ }^{1)-6)}$, and several theoretical mechanisms have also been presented. According to Robertson ${ }^{7}$, under the shear stress the structure of the glass is changed into a melt-like structure and on this condition the yield point appears, i.e., the glass begins to flow. Rendell, Ngai, Yee, and others ${ }^{8)}$ applied their "coupling model of relaxation" to nonlinear viscoelasticity to obtain constitutive equations including a time-dependent term, which was a modification of the Boltzmann principle. Using these constitutive equations they predicted successfully the stress response at constant strain rates, stepwise strain rates and stress relaxation for polycarbonate. Shay and Caruthers ${ }^{9}$ ) proposed a free-volume model for the yielding taking an effect of the deformation-induced time-dependent dilation into account. In contrast to these theories in which homogeneous change in the polymeric structure was assumed, Lefebvre and Escaig $^{10)}$ proposed the contribution of nucleation of molecular defects to the plastic strain, and suggested the inhomogeneous structural change during the yielding. Yet, in spite of the proposition of these theories, there was little experimental evidence verifying the structural transition to melt-like structures of the glass.

In the present paper, first, our experimental analyses convincingly verifying the structural transition to melt-like structures in glassy polymers are reviewed. The steady plastic flow in the post-yield state was analyzed using the Eyring equation in a novel way. This enabled us to find unique functional relations among the Eyring parameters; activation energy, activation entropy and activation volume. It was shown further that the functional relation between the activation energy and activation entropy for the glass agreed well with that determined for the equilibrium melt of the polymer. This agreement was evidence of the structural transition in the glass to the melt-like structure.

Secondly, our work on the aging behavior in glassy polymers subjected to plastic strain is reviewed. Structural relaxation due to aging in non-equilibrium melt-like structures was demonstrated using data of time-dependent mechanical property, differential scanning calorimetry and thermally stimulated deformation recovery. It was found that the strain energy given to glassy polymers during their yielding process was locked in tightly due to structural relaxation of the nonequilibrium structure.

Finally, a study on cold working of glassy polymers is presented. Applicability of the non-equilibrium melt-like structure in polymer glasses to their cold working is discussed.

" Present address: 310-202, 1-3, Fujisawadai, Tondabayashi, Osaka 584-0071, Japan 


\section{NONLINEAR PLASTIC DEFORMATION AND PLASTIC FLOW IN GLASSY POLYMERS}

\subsection{Plastic Deformation and Steady Plastic Flow}

As one of experimental approaches with regard to molecular kinetics of plastic deformation in glassy polymers, rate analyses on experimental data of the yield point (a point of peak stress) have been carried out by many workers. ${ }^{1)-6)}$ The yield point was regarded as a state of plastic flow, and it was frequently analyzed by using the Eyring equation. The analyses presumably postulated that the yield point was in the state of steady flow, since the Eyring equation ${ }^{11,12)}$ should solely be applied to the "steady state" of chemical and physical reaction processes. With regard to the yield flow, however, we found the stress at the yield point was changeable in magnitude depending on the strain rate history. ${ }^{13)}$ An example showing such changeable behavior is shown in Fig. 1. The stress responses in this figure were obtained by stepwise stretching of fully annealed poly(methyl methacrylate) (PMMA) specimens: the strain rate was increased stepwise from a value of $1.67 \times 10^{-6}\left(\mathrm{~s}^{-1}\right)$ to a higher value of $1.67 \times 10^{-4}\left(\mathrm{~s}^{-1}\right)$ at several levels of nominal strain $\varepsilon_{\mathrm{n}}$. The stress responses showed that the yield stress was considerably enhanced after a sudden increase of strain rate. Thus the value of the yield stress is not uniquely determined by the strain rate but depends on the strain rate history. This probably means that the yield point is an unstable state of flow, to which the application of the Eyring equation may be limited or unsuccessful.

By contrast, in the post-yield range of Fig. 1, most of the stress-strain curves converged to the same minimum value of stress in spite of the distinct difference in strain-rate history. It

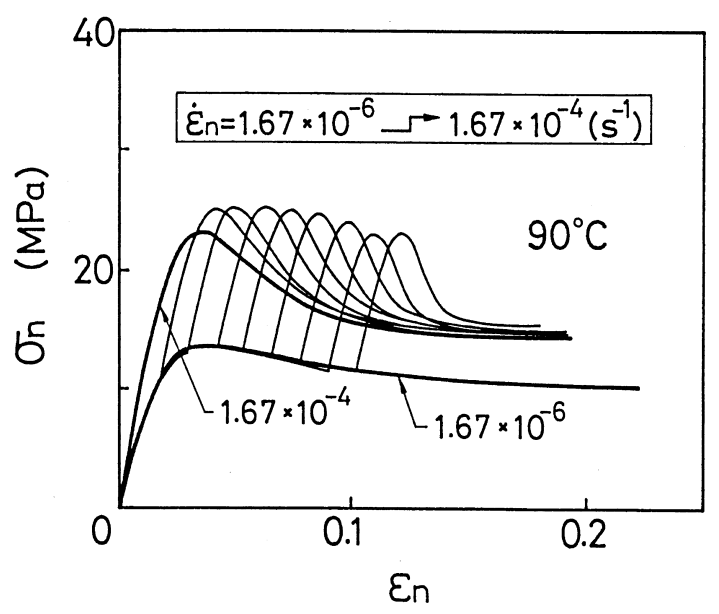

Fig. 1. Transient stress response for PMMA obtained by a sudden increase of stretching rate from $1.67 \times 10^{-6}$ to $1.67 \times 10^{-4}\left(\mathrm{~s}^{-1}\right)$. is also seen that this post-yield stable state reserved for a relatively much longer duration of strain compared to the instantaneous appearance of the yield point. Accordingly, it is likely to be much more reasonable that the post-yield flow range can be analyzed by the Eyring equation as a state of steady plastic flow.

\subsection{Rate Process of Post-Yield Plastic Flow}

The Eyring equation for shear viscous flow is given as

$$
\dot{\gamma}=\frac{2 \lambda k T}{\lambda_{1} h} \exp \left(-\frac{\Delta G}{R T}\right) \sinh \left(\frac{\tau v_{s}}{2 k T}\right)
$$

where $\dot{\gamma}$ is shear strain rate, $\tau$ is shear stress, $T$ the absolute temperature, $\Delta G$ the activation free energy, $v_{\mathrm{s}}$ the shear activation volume, $R$ the gas constant, $h$ Planck's constant, $k$ Bolzmann's constant, and $\lambda_{1}$ and $\lambda$ are distances between adjacent flow units neighboring in the vertical and parallel to the flow plane, respectively.

When a hydrostatic pressure component $p$ is imposed in addition to the shear stress $\tau$, Eq. (1) is modified as

$$
\dot{\gamma}=\frac{2 \lambda k T}{\lambda_{1} h} \exp \left(-\frac{\Delta G+p \Delta V}{R T}\right) \sinh \left(\frac{\tau v_{s}}{2 k T}\right)
$$

where $\Delta V$ refers to pressure activation volume per mole of flow units.

Substitution of Eqs. (3) and (4) below into Eq. (2) makes the Eyring equation applicable to a state of uniaxial flow under tensile stress.

$$
\tau=\frac{\sigma}{2}, \quad \dot{\gamma}=\frac{3}{2} \dot{\varepsilon}, \quad p=\frac{1}{3} \sigma
$$

and

$$
\Delta G=\Delta H-T \Delta S
$$

where $\sigma$ is uniaxial tensile stress, $\dot{\varepsilon}$ tensile strain rate, $\Delta H$ the activation energy and $\Delta S$ the activation entropy.

Using further the following approximate relation for high values of stress $\tau$ in such cases as plastic flow

$$
\sinh \left(\frac{\tau v_{s}}{2 k T}\right) \cong \frac{1}{2} \exp \left(\frac{\tau v_{s}}{2 k T}\right)
$$

we obtain an expression

$$
\frac{\sigma}{T}=\frac{9.212 k}{v_{t}}\left(\log \dot{\varepsilon}-\log C T+0.4343 \frac{\Delta H}{R T}\right)
$$


where

$$
v_{t}=v_{s}+\frac{4}{3} \Delta v
$$

and

$$
C=\frac{2 \lambda k}{3 \lambda_{1} h} \exp \left(\frac{\Delta S}{R}\right)
$$

The quantity $\Delta v$ in Eq. (7) designates pressure activation volume per single flow unit. The volume $v_{\mathrm{t}}$ in Eq. (6) is hence an apparent shear activation volume for the flow under tensile stress. Equation (6) predicts a linear relationship between $\sigma / T$ and $\log \dot{\varepsilon}$. For fully annealed PMMA specimens subjected to constant-rate stretching, Fig. 2 gives experimental relationship between the post-yield plastic flow stress $\sigma_{1}$ divided by temperature $T$ and the logarithm of true strain rate $\dot{\varepsilon}$ at various temperatures. The stress $\sigma_{1}$ is a true stress calculated on assumptions of uniform deformation and constant volume of the specimens during the uniaxial stretching. An approximate curve through the experimental points of $\sigma_{1} / T$ against $\log \dot{\varepsilon}$ at each temperature shows a non-linear functional relation. Hence the linear equation (6) cannot be fitted to any of these experimental curves if the Eyring parameters $\Delta H, v_{\mathrm{t}}$ and $C$ are assumed to be constant through the experimental range of strain rate. The constancy of the Eyring parameters, however, leads us to be suspicious of the restriction that the molecular mechanism of an elementary process of flow in a glassy polymer must always be unchangeable independent of

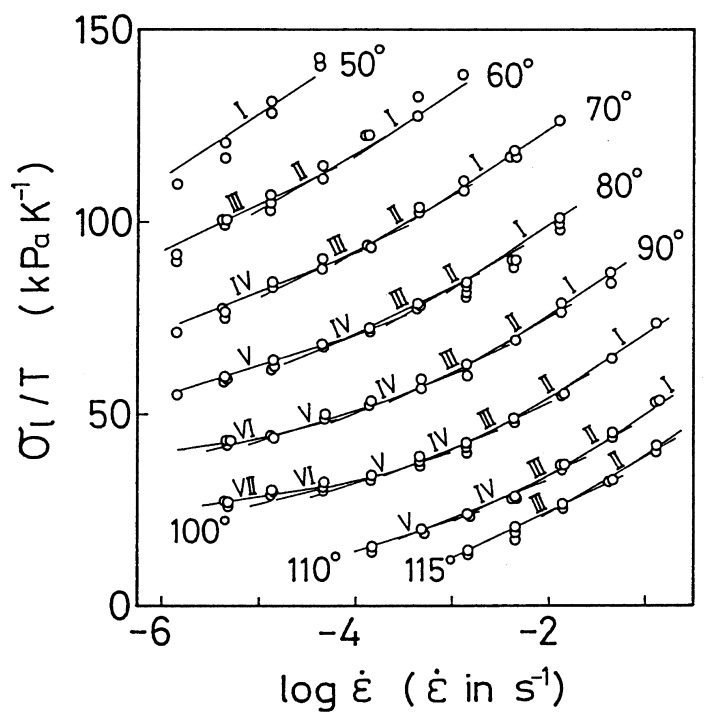

Fig. 2. Ratio of post-yield flow stress $\sigma_{1}$ to temperature $T$ plotted against the logarithm of tensile rate $\dot{\varepsilon}$ for PMMA. Sets of parallel line elements were obtained by fitting Eq. (6) best to the experimental data. strain rate, stress or temperature. But as an evidential example for a molecular process influenced by these experimental parameters, the activation energy for the relaxation process in a polymer melt near the glass transition temperature $T_{\mathrm{g}}$ has been observed to be strongly temperature dependent. Accordingly, the non-linear curve of $\sigma_{1} / T$ against $\log \dot{\varepsilon}$ requires another approach for rate-process analysis.

One such approach may be a use of the Ree-Eyring equation ${ }^{14)}$ for multiple stages as already demonstrated by Roetling ${ }^{1)}$ and others. The application of this equation to plastic flow yet limits the number of elementary processes in plastic flow to the number of the multiple stages used. If the structure of the polymer glass in the post-yield range is continuously changeable depending on strain-rate and temperature, as similar to the relaxation behavior of the melt at temperatures near $T_{\mathrm{g}}$, then the Ree-Eyring equation may not be adequate for the analysis of the plastic flow. In such a case, the Eyring parameters must be treated as continuously changeable in accordance with the structural change. In consequence, it seems quite reasonable that the Eyring parameters $\Delta H, v_{\mathrm{t}}$ and $C$ in Eq. (6) should be treated here as continuous variables dependent on strain rate and temperature. Experimental values of these Eyring parameters for the data shown in Fig. 2 could be determined in the following manner.

Each nonlinear curve through the experimental plots in Fig. 2 was replaced by a chain of elemental lines. A set of parallel elemental lines was so calculated as to fit Eq. (6) best with the experimental plots and is shown in Fig. 2. Roman numerals labeling each line element correspond to experimentally evaluated Eyring parameters in Table I labeled by the same numerals. This novel procedure for evaluating the Eyring parameters will subsequently be proved successful also for other glassy polymers; polyvinylchloride(PVC), polycarbonate (PC) and polystyrene(PS).

Table I Numerical values of Eyring parameters obtained by applying Eq. (6) to post-yield steady plastic flow in poly(methyl methacrylate) in constant rate tension.

\begin{tabular}{ccrr}
\hline \hline Symbo1 & $\begin{array}{c}\Delta H \\
(\mathrm{~kJ} / \mathrm{mo1})\end{array}$ & $\log C$ & $\begin{array}{c}v_{\mathrm{t}} \\
\left(\mathrm{nm}^{3}\right)\end{array}$ \\
\hline I & 343 & 40.21 & 7.70 \\
II & 377 & 44.45 & 8.76 \\
III & 431 & 51.38 & 10.60 \\
IV & 494 & 59.49 & 12.72 \\
V & 607 & 74.40 & 15.88 \\
VI & 737 & 91.14 & 21.18 \\
VII & 1005 & 125.96 & 31.78 \\
\hline
\end{tabular}


The parameter $\log C$ in Table I is approximately convertible into the activation entropy $\Delta S$ in the following manner. From Eq. (8),

$$
\log C=0.4343 \frac{\Delta S}{R}+\log \left(\frac{2 \lambda_{k}}{3 \lambda_{1} h}\right)
$$

In this equation, it may be assumed that the quantities $\lambda_{1}$ and $\lambda$ are of the same order of magnitude. Then, we have

$$
\Delta S=19.15(\log C-10.14)
$$

Substitution of the experimental values of $\log C$ shown in Table I into this equation allowed us to obtain approximate experimental values of the activation entropy $\Delta S$ for the plastic flow in glassy polymers.

Experimental values of $\Delta S$ thus obtained are plotted against the activation volume $v_{\mathrm{t}}$ in Fig. 3 , where the experimental values of $\Delta H$ are also plotted against the same horizontal axis $v_{\mathrm{t}}$. In this figure the symbols $\Delta S$ and $\Delta H$ have been replaced with $\Delta S^{(\mathrm{g})}$ and $\Delta H^{(\mathrm{g})}$, respectively, to define them explicitly as obtained from the glassy state. It is seen that both $\Delta S^{(\mathrm{g})}$ and $\Delta H^{(\mathrm{g})}$ increase linearly with $v_{\mathrm{t}}$.

The activation entropy $\Delta S^{(\mathrm{g})}$ represents the change in the order of molecular arrangement in flow units presumably occupying the volume $v_{\mathrm{t}}$ when it is activated by the activation energy $\Delta H^{(\mathrm{g})}$. Hence, the experimental value of $\Delta S^{(\mathrm{g})}$, always positive as seen in Fig. 3, implies that the structural order of

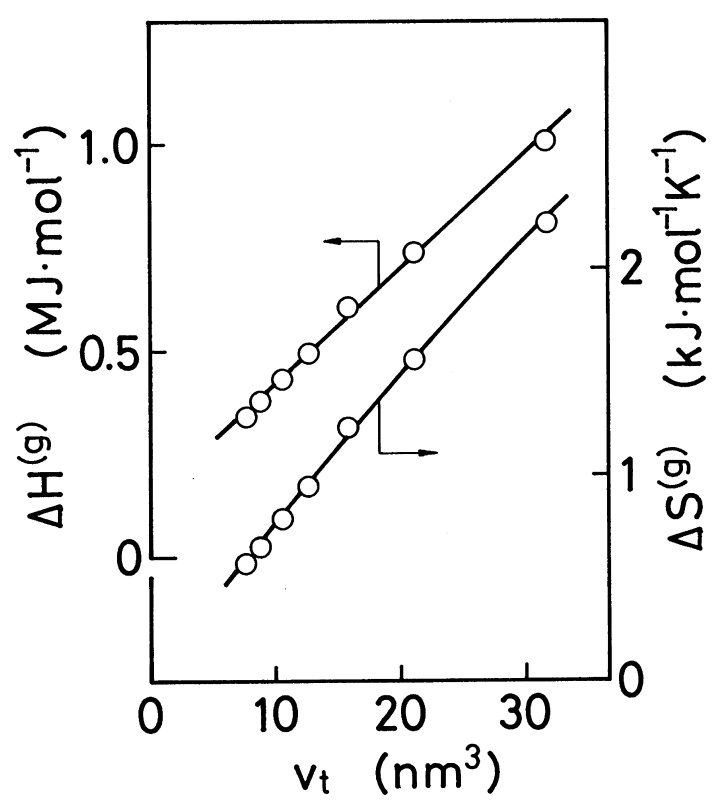

Fig. 3. Activation energy $\Delta H^{(\mathrm{g})}$ and activation entropy $\Delta S^{(\mathrm{g})}$ plotted against apparent activation volume $v_{\mathrm{t}}$ for steady plastic flow in PMMA glass under tensile stress. molecules in a flow unit lessens at all times when it jumps over a potential energy barrier. This suggests that in an activated flow unit the inter- and intra-molecular forces may be comparatively loosened.

In connection with the Eyring parameters obtained above for the plastic flow in glassy PMMA, let us notice the viscous flow in the melt of the same PMMA sample from the viewpoint of the activation process. As shown subsequently, this will enable us to advance discussions several steps forward concerning the physical meanings of the Eyring parameters controlling the molecular kinetics of the plastic flow in glassy polymers.

Concerning the activation processes in the states of glass and melt, intensive attention will be paid to their relationship between $\Delta S$ and $\Delta H$. The relation of $\Delta S^{(\mathrm{g})}$ and $\Delta H^{(\mathrm{g})}$ could be derived from the relation of $\Delta H^{(\mathrm{g})}$ to $\log C$ as shown above. The relation of $\Delta S$ to $\Delta H$ for the melt, on the other hand, can be derived from linear viscoelastic relaxation data by using the thermodynamics theory in the following way. The expression of the activation energy for the steady flow of a polymer melt $\Delta H^{(\mathrm{m})}$ can be obtained from the WLF equation as

$$
\Delta H^{(m)}=\frac{2.303 R c_{1}^{g} c_{2}^{g} T^{2}}{\left(T-T_{g}+c_{2}^{g}\right)^{2}}
$$

where $c_{1}{ }^{\mathrm{g}}$ and $c_{2}{ }^{\mathrm{g}}$ are the WLF parameters evaluated at a reference temperature $T_{\mathrm{g}}$ (the glass transition temperature). Numerical values of $T_{\mathrm{g}}$ and the WLF parameters for the PMMA sample used in this study were determined by a stress relaxation experiment in a manner described elsewhere: ${ }^{15)}$

$$
T_{\mathrm{g}}=389 \mathrm{~K}, c_{1}{ }^{\mathrm{g}}=21.2 \text { and }{c_{2}}^{\mathrm{g}}=66.4 \text {. }
$$

Since the activation energy $\Delta H^{(\mathrm{m})}$ is given as a function of temperature $T$ as seen in Eq. (11), the expression of the activation entropy for the molten polymer $\Delta S^{(\mathrm{m})}$ can be deduced from Eq. (11):

$$
\Delta S^{(m)}=\frac{2.303 R c_{1}{ }^{g} c_{2}{ }^{g}\left(T_{g}-c_{2}{ }^{g}\right)}{\left(T-T_{g}+c_{2}^{g}\right)^{2}}
$$

Thus, from Eqs. (11) and (12) using the numerical values for the parameters $T_{\mathrm{g}}, c_{1}{ }^{\mathrm{g}}$ and $c_{2}{ }^{\mathrm{g}}$, the relation of $\Delta S^{(\mathrm{m})}$ to $\Delta H^{(\mathrm{m})}$ is expressed as

$$
\sqrt{\Delta H^{(m)}}=17.96 \sqrt{\Delta S^{(m)}}+5.19
$$

where $\Delta S^{(\mathrm{m})}$ is presented in $\mathrm{kJ} / \mathrm{mol} \cdot \mathrm{K}$ and $\Delta H^{(\mathrm{m})}$ in $\mathrm{kJ} / \mathrm{mol}$.

In Fig.4, the relation of $\Delta S^{(\mathrm{g})}$ to $\Delta H^{(\mathrm{g})}$ calculated from the data in Table I is shown and compared with a solid line 
representing Eq. (13). As evident from this comparison, the two relations are in good agreement. Namely, the two entropies $\Delta S^{(\mathrm{m})}$ and $\Delta S^{(\mathrm{g})}$ are about the same in magnitude for any value of $\Delta H$. This implies that in either the glassy state or the melt the structural disorder in flow units increases by the same amount $\Delta S$ when they jump over a potential energy barrier as they are activated by the energy $\Delta H$. Hence, it is probable that the elementary process of molecular flow in the glass is identical with that in the melt although the temperature of the glass is far lower than that of the melt. This agreement requires that the amorphous structure of the glass must be equivalent to that of the melt. In other words, the structure of the glass is isothermally changed into the structure of equilibrium melt by the imposition of plastic flow strain.

What can be equivalent in the structures of the flowing glass and the equilibrium melt? The quantity $\Delta H$ is the energy required for a molecular segment or a cluster of segments (i.e., a flow unit) to pass over the potential energy barrier. In other words, being activated by the energy $\Delta H$ and also aided by stress, a flow unit makes a single displacement step. This movement occurs under the influence of the intermolecular interaction between the flow unit and its surrounding molecular segments. Thus, the value of $\Delta H$ is determined by the intermolecular interaction. Another activation parameter $\Delta S$ is a measure of the structural disorder occurring in a flow unit when passing over the barrier. Hence the value of $\Delta S$ is also controlled by the intermolecular interaction. Accordingly, it is most probable that what is equivalent in the flowing glass and the equilibrium melt is the intermolecular interaction.

The intermolecular interaction in polymeric systems is determined by various secondary molecular forces, such as hydrogen bonding, dipole interactions, van der Waals forces and steric hindrance. Hence, in the flowing polymer glass, these secondary interactions have presumably been lessened by imposed stress or strain compared with those in the undeformed metastable glassy state, and the intermolecular interaction in the glass has become equivalent to that in the equilibrium melt.

Since the amorphous structure being changed into that of the equilibrium melt is expected also in the state of plastic flow in other glassy polymers, the post-yield plastic flow observed for PVC, PC and PS was analyzed by the same novel approach as used for PMMA. ${ }^{16,17)}$ The results are summarized in Fig. 5 where the ordinates are arbitrarily given a vertical shift of even interval. Open circles are the data obtained from the steady plastic flow of glassy polymers and solid lines were derived from the relaxation data of the polymer melts. From this figure one can be convinced that the structural transition into the structure of the equilibrium melt is generally induced by constant rate stretching of glassy polymers in their post-yield steady flow state.

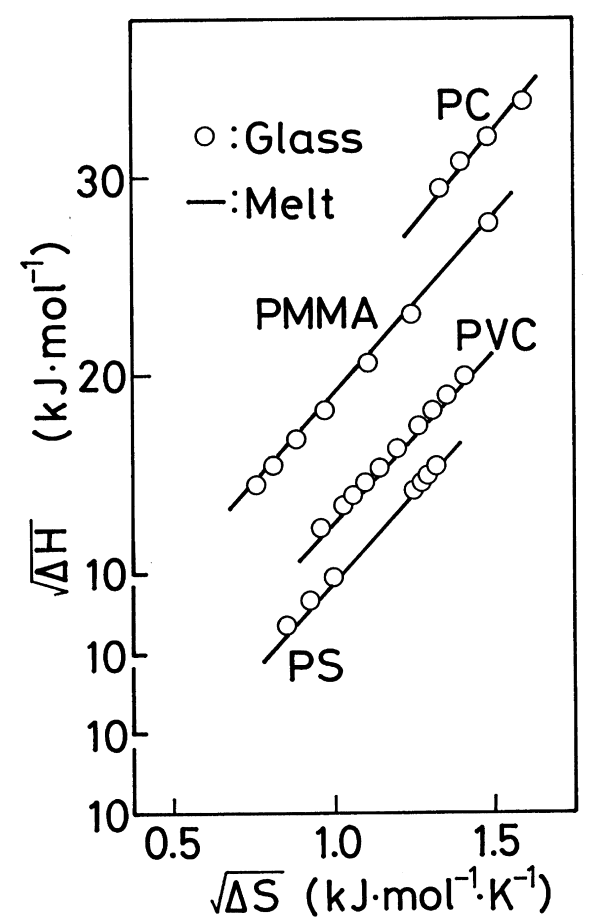

Fig. 5. Square-root plots of $\Delta H$ and $\Delta S$ of the glass in plastic flow and the melt in equilibrium for PMMA, PVC, PC and PS. of activation entropy $\Delta S$ for steady plastic flow in PMMA glass, compared with square-root plot of $\Delta H$ and $\Delta S$ for the same PMMA sample in the state of equilibrium melt. 


\subsection{Effect of Compressive Stress on the Isothermal Structural Transition}

The post-yield steady plastic flow is observed also in constant rate compression of glassy polymers. In this section the same novel approach of rate analysis as used above is applied to the post-yield plastic flow under compressive stress for the same PMMA sample as was used in the stretching test. Besides verifying the structural transition into the melt structure under compression, this analysis enables us to evaluate the contribution of the hydrostatic stress component to the flow in the glass.

In the Eyring equation of the form of Eq. (6), the quantity $v_{t}$ should be replaced with $v_{\mathrm{c}}$ (apparent shear activation volume in compression) for the rate analysis of compression data;

$$
\frac{\sigma}{T}=\frac{9.212 k}{v_{c}}\left(\log \dot{\varepsilon}-\log C T+0.4343 \frac{\Delta H}{R T}\right)
$$

where

$$
v_{c}=v_{s}-\frac{4}{3} \Delta v
$$

The plot of $\sigma_{1} / T$ against $\log \dot{\varepsilon}$ for constant rate uniaxial compression of the PMMA sample is shown in Fig. $6 .{ }^{18)}$ Line elements I to X were obtained by best fitting Eq. (14) to the

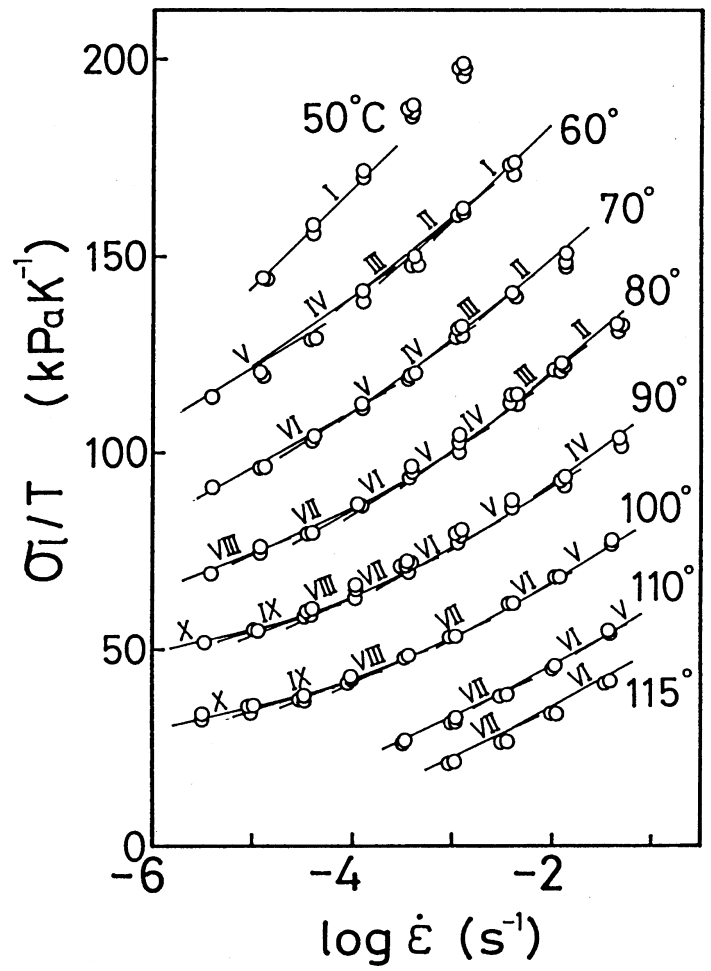

Fig. 6. Ratio of post-yield flow plastic stress $\sigma_{1}$ in compression to $T$ plotted against $\log \dot{\varepsilon}$ for the same PMMA sample as used in the stretching test. data, giving numerical values of the Eyring parameters $\Delta H, C$ and $v_{c}$. The quantity $C$ was converted to $\Delta S$ using Eq. (10). In Fig. $7,{ }^{19)}$ the experimental relation between $\Delta H$ and $\Delta S$ in square-root for the glass in uniaxial compression is compared with Eq. (13) expressing the relation of $\Delta H$ to $\Delta S$ for the melt. An excellent agreement of the two functional relations is seen, thus providing evidence of the glass-to-melt structural transition in the glass in uniaxial compression.

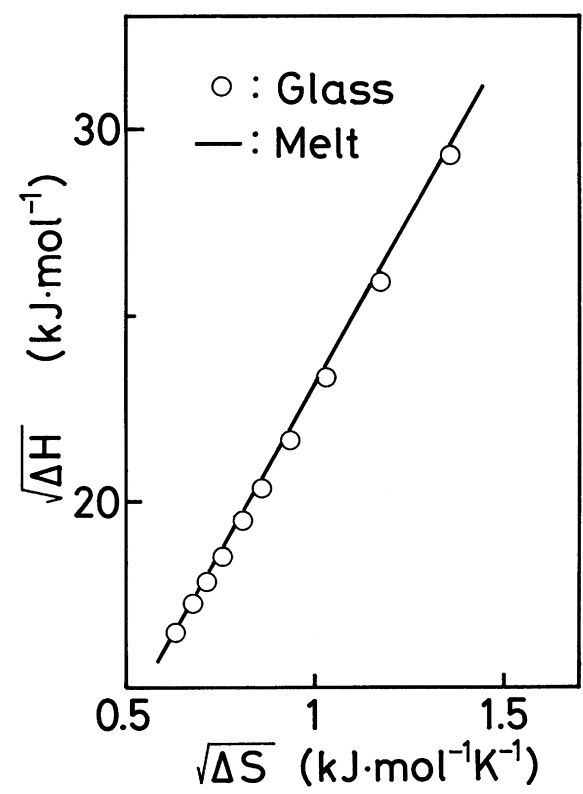

Fig. 7. Square-root plot of $\Delta H$ against $\Delta S$ for PMMA glass in compression compared with that for the equilibrium melt of the same PMMA sample.

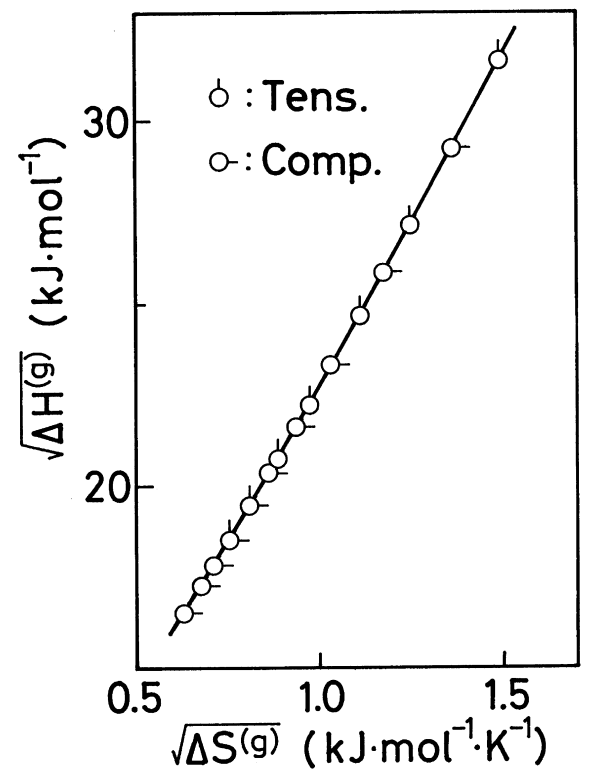

Fig. 8. Comparison of square-root plots of $\Delta H^{(\mathrm{g})}$ against $\Delta S^{(\mathrm{g})}$ obtained for the same PMMA sample in tension and in compression. All the experimental points fall on a single curve. 
Furthermore, the comparison of the compression data with the tension data both obtained from the same sample can be worthwhile for evaluating the effect of the hydrostatic component on the structural transition. The relation of $\Delta H$ against $\Delta S$ from the compression test is plotted in square-root together with that from the tension test in Fig. $8 .{ }^{19)}$ It is found that both the relations are represented by a single curve. This single functional relation between $\Delta H$ and $\Delta S$ presumably expresses a characteristic equation of the melt-like structure of the glass, which is not influenced by the hydrostatic component.

On the other hand, the product $p \Delta V$ in Eq. (2) is an energy term representing the influence of the hydrostatic component $p$ on the flow kinetics of the melt-like structure. Hence, if the value of $\Delta V$ (or $\Delta v$, which is equal to $\Delta V$ divided by the Avogadro number) can be determined, this provides a quantitative estimate of the influence of $p$ on the flow kinetics in the glass.

In Fig. 9, the apparent shear activation volumes $v_{\mathrm{t}}$ and $v_{\mathrm{c}}$ are plotted against the activation energy $\Delta H$, the value of which is a quantitative measure representing a state of the melt-like structure. This plot shows that the volume $v_{\mathrm{t}}$ and $v_{\mathrm{c}}$ are unique functions of $\Delta H$ and the value of $v_{\mathrm{t}}$ is always larger than that of $v_{\mathrm{c}}$ for any value of $\Delta H$. Hence, we can derive the equation of $v_{\mathrm{s}}$ and $\Delta v$ from Eqs. (7) and (15):

$$
v_{s}=\frac{1}{2}\left(v_{t}+v_{c}\right)
$$

and

$$
\Delta v=\frac{3}{8}\left(v_{t}-v_{c}\right)
$$

where $\Delta v$ is the pressure activation volume per flow unit. Substitution of the numerical data shown in Fig. 9 into these equations provides plots of $v_{\mathrm{s}}$ and $\Delta v$ as a function of $\Delta H$. These are shown in Fig. 10, where the scale of $\Delta v$ is magnified by a factor of 10 .

The positive numerical value of $\Delta v$ should be noticed. This indicates that the volume of a flow unit $v_{\mathrm{s}}$ always increases by an amount $\Delta v$ when it passes over the potential energy barrier with the aid of shear stress. The increase in volume is independent of the sign of the imposed pressure component. This is similar to the fact that the experimental values of the activation entropy $\Delta S$ are always positive regardless of the sign of the pressure component. This correspondence is reasonable because the order of the molecular segments in an activated flow unit is decreased by the amount $\Delta S$, probably due to weakening of intermolecular forces by conformational rearrangement under the influence of local excess shear strain. At this moment, the flow unit simultaneously increases in volume by an amount $\Delta v$ due to the probable decrease of intermolecular forces.

As for the amount of $\Delta v$ (the amount of volume increase in an activated flow unit), the ratio of $\Delta v$ to $v_{\mathrm{s}}$ is given in Fig. 10 as a function of $\Delta H$. The ratio increased with $\Delta H$, showing a value of approximately 4 to $7 \%$ of the shear activation volume $v_{\mathrm{s}}$.

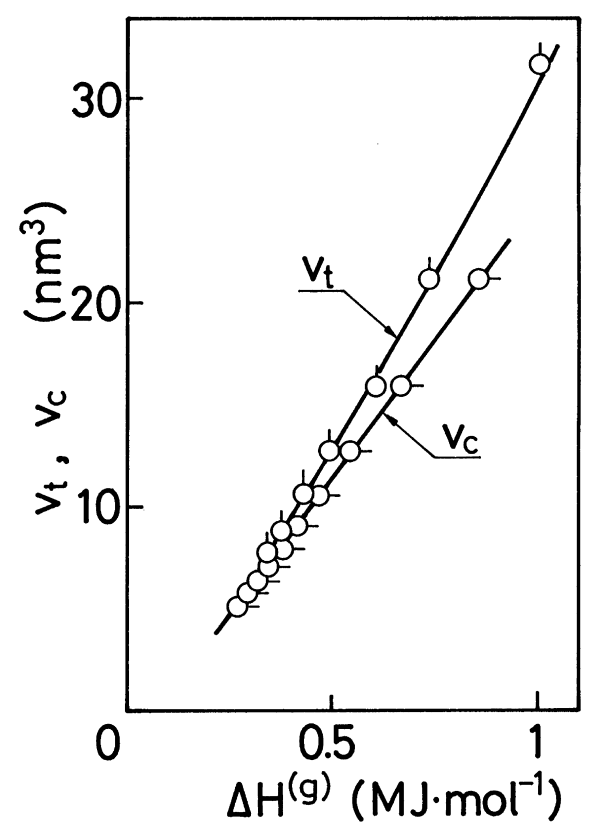

Fig. 9. Plots of apparent shear activation volume in tension and in compression, $v_{\mathrm{t}}$ and $v_{\mathrm{c}}$ respectively, against activation energy $\Delta H^{(\mathrm{g})}$

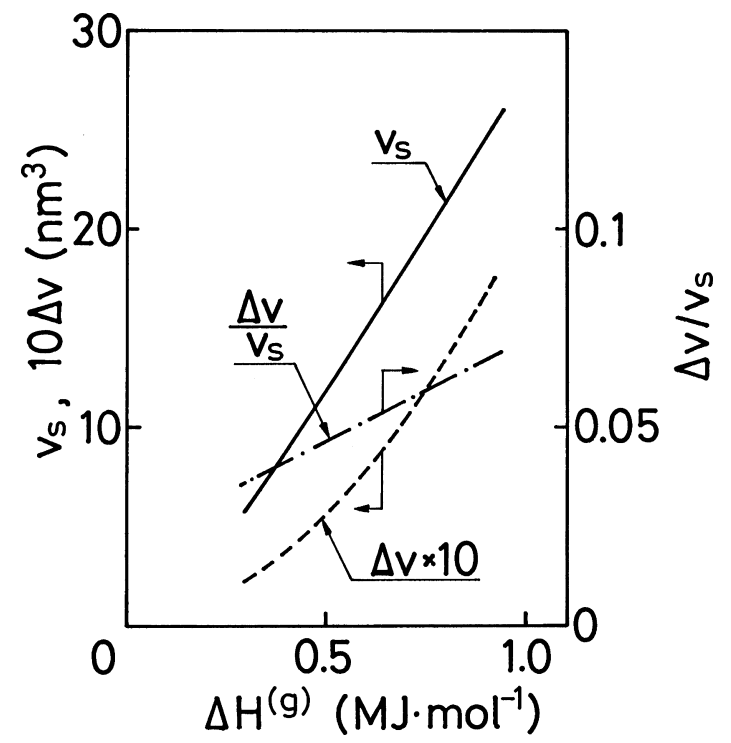

Fig. 10. Numerical values of quantities $v_{\mathrm{s}}$ and $\Delta v$, and ratio of $\Delta v$ to $v_{\mathrm{s}}$ as a function of $\Delta H^{(\mathrm{g})}$. 


\subsection{Isothermal Glass-to-Melt Structural Transition at Low Temperatures}

Our experimental evidence for the glass-to-melt structural transition in glassy polymers has so far been obtained at relatively high temperatures below $T_{\mathrm{g}}$, at which the microBrownian motion of molecular segments is believed to be still probable. It might therefore be suspected that the local segmental motion in the glass makes some contribution to the plastic deformation. Thus in the present section, the flow of the same PMMA sample as used above at moderate temperatures is examined in a temperature range below its equilibrium second-order transition temperature $T_{2}{ }^{20)}$ In this temperature range, molecular configurational change in the polymer main chain is supposed to be completely prohibited, as referred to the state of zero configurational entropy. The analysis enables us to correlate the plastic flow mechanism in the lower temperature range with that in the higher temperature range; at temperatures below $T_{2}$, the structure of the glass is also changed into melt-like structures.

Figure $11^{21)}$ shows plots of true stress against nominal strain ( $\sigma$ against $\varepsilon_{\mathrm{n}}$ ) for cylindrical specimens of poly(methyl methacrylate) (PMMA) at various temperatures from $50{ }^{\circ} \mathrm{C}$ to $-50{ }^{\circ} \mathrm{C}$ obtained by a constant rate uniaxial compression at a strain rate of $3.13 \times 10^{-5}\left(\mathrm{~s}^{-1}\right)$. The true stress $\sigma$ was calculated on assumptions of uniform deformation and constant volume of the specimens during the uniaxial compression. Passing through the yield point, the true stress then decreased to a

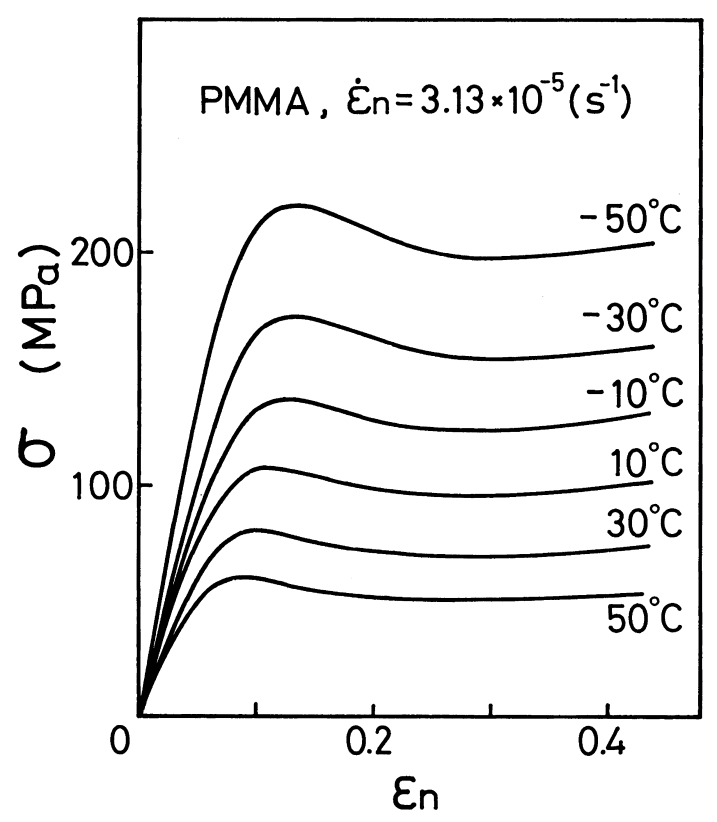

Fig. 11. Stress strain relations for PMMA in constant-rate compression at temperatures far below $T_{\mathrm{g}}$. minimum value in a post-yield plateau range. As stated above, this post-yield range can reasonably be considered as a state of steady plastic flow in the glassy polymer, to which the Eyring equation is convincingly applicable.

Experimental data of the post-yield flow stress $\sigma_{1}$ divided by temperature $T$ over a temperature range from $-40{ }^{\circ} \mathrm{C}$ to $+60^{\circ} \mathrm{C}$ are plotted against $\log \dot{\varepsilon}$ in Fig. 12. ${ }^{21)}$ The plot can be analyzed using the Eyring equation (14) in such a novel way as illustrated above. Sets of parallel line elements labeled by alphabetical marks $a$ through $k$ in Fig. 12 were obtained by fitting Eq. (14) best to the plot and corresponding numerical values of the Eyring parameters $\Delta H, v_{\mathrm{c}}$ and $C$ were determined. The quantity $C$ is converted to $\Delta S$ using Eq. (10).

In Fig. 13, the functional relation of $\Delta H$ and $\Delta S$ obtained for the glass is compared with that for the equilibrium melt, i.e., a dashed straight line as indicated by "WLF equation". Filled circles are the data of the glass obtained at relatively high temperatures and open circles are of the glass at lower temperatures. The experimental points for the glass at relatively high temperatures are in good agreement with the line of WLF equation as already stated, whereas in the lower temperature range experimental points deviate gradually from the straight line of WLF equation with decreasing $\Delta H$.

A likely explanation for this deviation is that the WLF equation in the range where the deviation occurs is a part of the

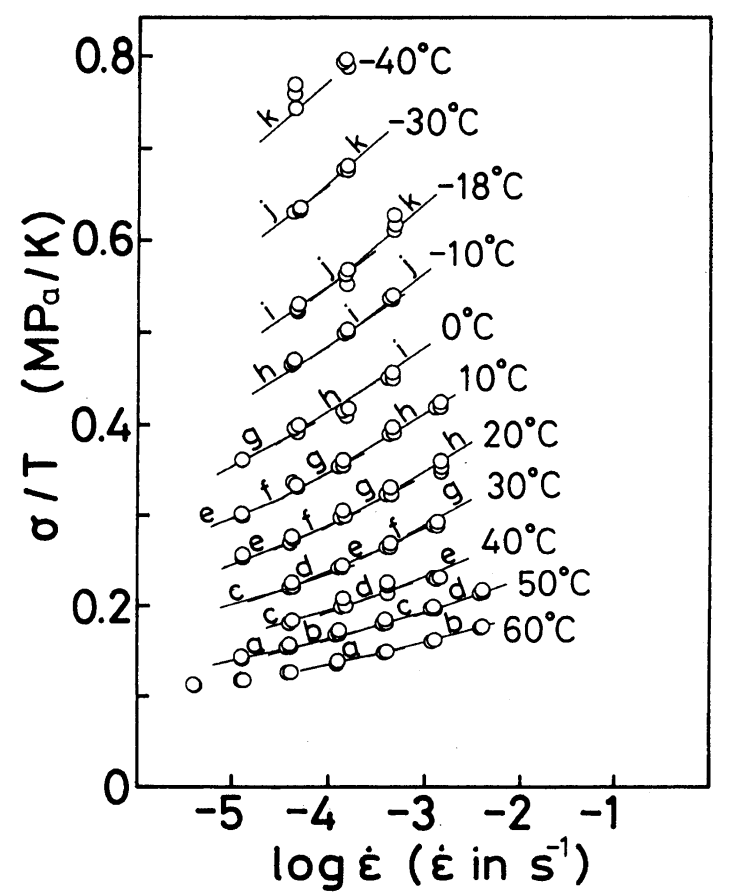

Fig. 12. Ratio of post-yield flow stress $\sigma_{1}$ to temperature $T$ plotted against the logarithm of true strain rate $\dot{\varepsilon}$ for PMMA deformed in constant-rate compression at temperatures far below $T_{\mathrm{g}}$. 
WLF equation extrapolated into a much higher temperature range where this empirical equation is no longer valid for the temperature dependence of relaxation. With respect to the temperature range of validity of the WLF equation it has been believed that the range from $T_{\mathrm{g}}$ to nearly $T_{\mathrm{g}}+100 \mathrm{~K}$ is acceptable, and at temperatures higher than this range the relaxation processes are governed by more specific features. ${ }^{22}$ Thus, if the structure of the glass is changed into a melt-like structure of the melt at higher temperatures beyond this temperature range, the relation between $\Delta H$ and $\Delta S$ for the melt-like structure may no longer be in agreement with the relation of $\Delta H$ to $\Delta S$ derived from the WLF equation. We are able to confirm this explanation by calculating the temperature of the melt from the quantity $\Delta S$. The quantity $\Delta S$ was converted to a corresponding temperature $T$ of the melt through the following equation:

$$
\Delta S=\frac{2.303 R c_{1}^{g} c_{2}^{g}\left(T_{g}-c_{2}^{g}\right)}{\left(T-T_{g}+c_{2}^{g}\right)^{2}}
$$

This calculation gives a second abscissa, $T-T_{\mathrm{g}}$, as shown in Fig. 12. It is noticeable that the experimental points of $\Delta H$

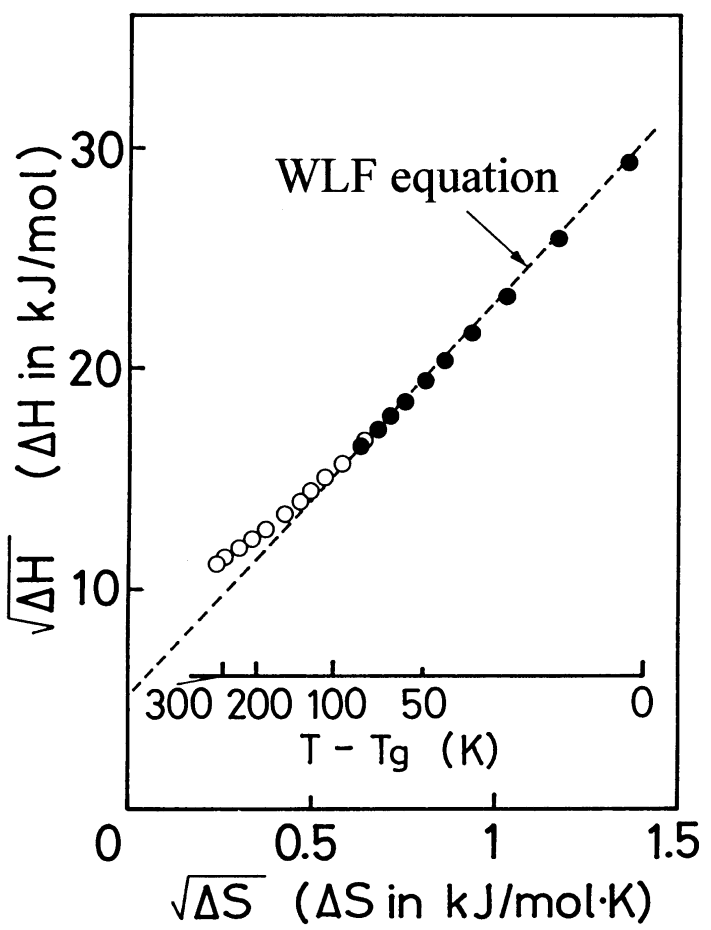

Fig. 13. Square-root of activation energy $\Delta H$ plotted against square-root of activation entropy $\Delta S$ for steady plastic flow in PMMA glass at low and high temperatures below $T_{\mathrm{g}}$, compared with square-root plot of $\Delta H$ and $\Delta S$ derived from the WLF equation for the same PMMA sample in the state of equilibrium melt. Symbols $\circ$ are the data obtained at lower temperatures and symbols $\bullet$ are at higher temperatures against $\Delta S$ of the glass begin to deviate from the line of WLF equation approximately at $T-T_{\mathrm{g}}=100 \mathrm{~K}$. Accordingly, the functional relation of $\Delta H$ and $\Delta S$ of the glass in the lower temperature range presumably corresponds to the relation of $\Delta H$ and $\Delta S$ of the melt at temperatures higher than $T_{\mathrm{g}}+100 \mathrm{~K}$, and the structure of the glass makes a transition into that of the melt at the higher temperatures. In consequence, comparison of the functional relations of $\Delta H$ and $\Delta S$ between the glass and the melt leads us to a suggestion that the glass is changed into a melt-like structure similar to that of the melt at very high temperatures when subjected to plastic deformations at very low temperatures.

Viscosity data of the PMMA melt can provide us with further confirmation on this glass-to-melt structural transition. For an injection-molding PMMA sample of moderate molecular weight, Asami ${ }^{23)}$ presented viscosity data as a function of temperature and strain rate over a temperature range from 200 to $260{ }^{\circ} \mathrm{C}$. Derived from his data, the experimental functional relation between the zero-shear viscosity $\eta_{0}$ and temperature $T$ for the PMMA sample is expressed by the following Arrhenius-type equation:

$$
\eta_{0}=A \exp \left(\frac{\Delta H^{(m)}}{R T}\right)
$$

where $\Delta H^{(\mathrm{m})}=146.5(\mathrm{~kJ} / \mathrm{mol})$ and $\ln A=-27.21$. The zeroshear viscosity equation for the melt-like structure of the PMMA glass can be predicted in the following way: The unapproximated original form of the Eyring equation is given as

$$
\dot{\gamma}=\frac{2 \lambda k T}{\lambda_{1} h} \exp \left(-\frac{\Delta H-T \Delta S+p \Delta V}{R T}\right) \sinh \left(\frac{\tau v_{s}}{2 k T}\right)
$$

This equation holds for a melt-like structure which is uniquely defined by numerical values of $\Delta H, \Delta S, v_{\mathrm{s}}$ and $\Delta V$. We suppose here this melt-like structure is to flow under very low stress $\tau$ without changing its structure (i.e., without changing the values of $\Delta H, \Delta S, v_{\mathrm{s}}$ and $\left.\Delta V\right)$, then we can derive an equation equivalent to the zero-shear viscosity for this structure from Eq. (20) as

$$
\eta_{0}{ }^{\prime}=\lim _{\tau, p \rightarrow 0} \frac{\tau}{\dot{\gamma}}=\frac{\lambda_{1} h}{\lambda v_{s}} \exp \left(-\frac{\Delta S}{R}\right) \exp \left(\frac{\Delta H}{R T}\right)
$$

In this expression $v_{\mathrm{s}}$ denotes shear activation volume, which has experimentally been shown to be related to the apparent 
activation volume $v_{c}$ evaluated in uniaxial compression in the following form:

$$
v_{c}=v_{s}-\frac{4}{3} \Delta v \cong 0.93 v_{s}
$$

Consequently, using experimentally determined parameters $v_{\mathrm{c}}$, $C$ and $\Delta H$ for the glass, Eq. (21) is expressed as

$$
\eta_{0}{ }^{\prime}=A^{\prime} \exp \left(\frac{\Delta H}{R T}\right)
$$

where

$$
A^{\prime} \cong 0.62 \frac{k}{C v_{c}}
$$

We now compare the experimental relation of $\ln A^{\prime}$ against $\Delta H$ of the melt-like glass with the point of $\ln A$ against $\Delta H$ of the melt from Asami's data. The result is shown in Fig. 14. The experimental point of $\ln A$ against $\Delta H$ (indicated by a filled square) falls on a straight line drawn through the data of $\ln A^{\prime}$ against $\Delta H$ of the melt-like glass. This agreement convincingly indicates the transition of the deformed low-temperature glass into a melt-like structure similar to that found in the melt at high temperatures. In consequence, it is concluded that through plastic deformation at a low temperature the glass

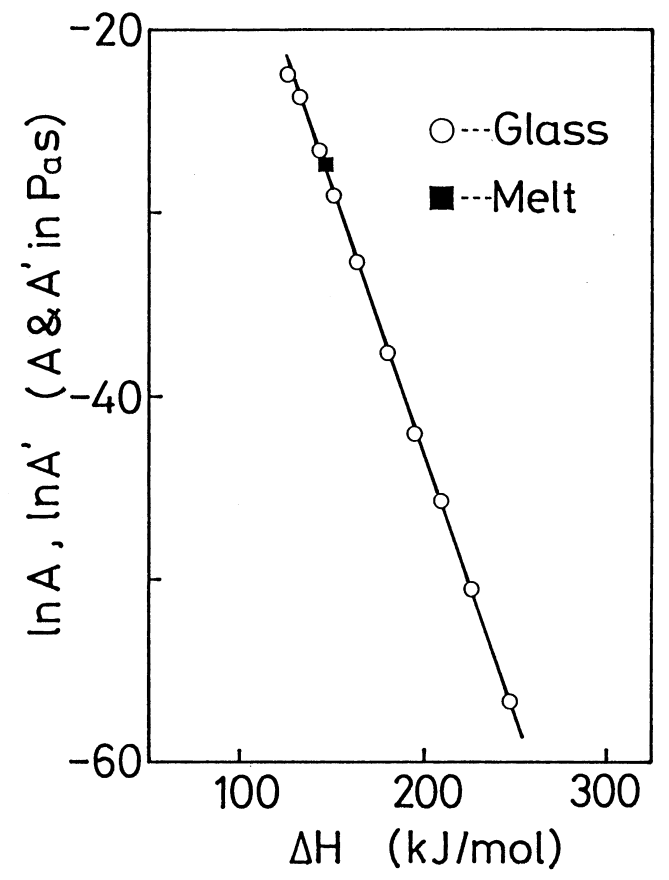

Fig. 14. Functional relation between zero-shear viscosity parameters $A^{\prime}$ and $\Delta H$ for the melt-like structures of PMMA glass, showing an excellent agreement with a plot of zero-shear parameter $A$ against $\Delta H$ for the equilibrium melt. changes its structure into a melt-like structure corresponding to that of the melt at much elevated temperatures. This is a structure likely to be similar to those in quenched nonequilibrium polymers.

\section{STRUCTURAL EVOLUTION IN GLASSY POLYMERS AGED UNDER PLASTIC STRAIN}

\subsection{Strain Aging in Annealed PMMA}

Polymer products are often subjected to plastic deformation as in cases when they are fastened tightly by steel bolts and nuts. At the moment of imposition of plastic strain, metastable structure of fully annealed amorphous polymers is changed to unstable melt-like ones as illustrated in the above section. In a polymer given plastic deformation, its non-equilibrium structure is expected to be changing toward some equilibrium structure. We will here refer to such structural relaxation phenomena as strain aging.

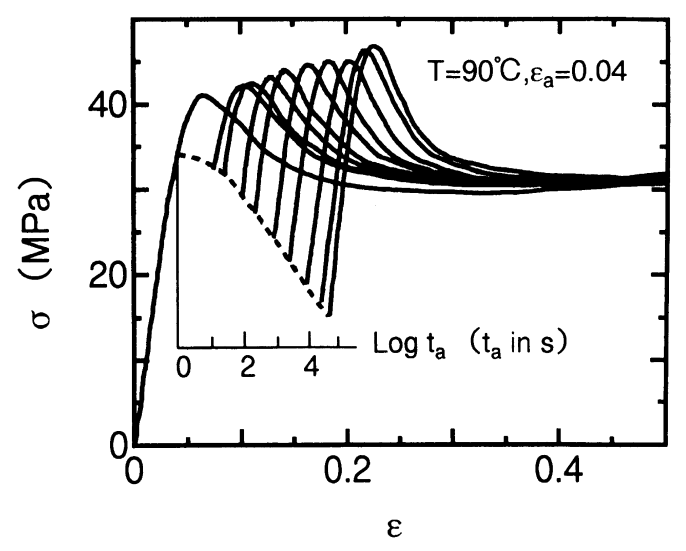

Fig. 15. Compressive true stress-strain relations for PMMA specimens aged under a compressive strain of 0.04 for various time periods $t$, showing evolution of yield stress with increasing aging time $t_{\mathrm{a}}$.

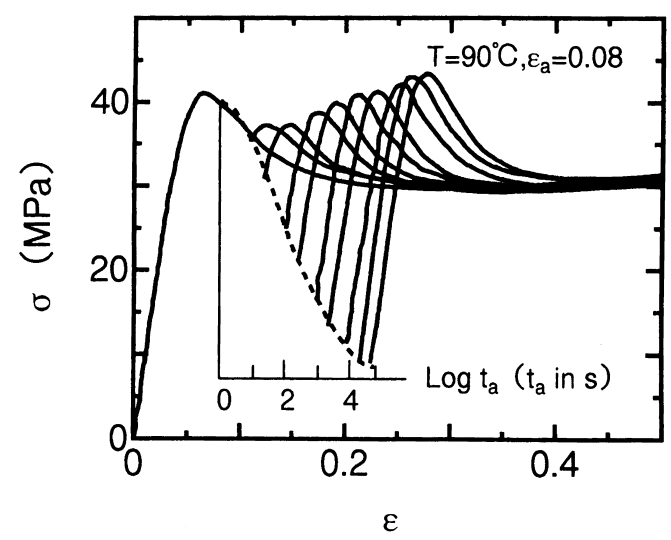

Fig. 16. Evolution of yield stress with aging time $t_{\mathrm{a}}$ in PMMA specimens aged under a compressive strain of 0.08 . 
Fully annealed PMMA specimens (hereafter simply referred to as annealed specimens) were strained by 0.04 in compression at $90^{\circ} \mathrm{C}$ and aged in the strained state at the same temperature for various time periods up to $24 \mathrm{~h}$. The compressive true stress-strain $(\sigma-\varepsilon)$ relations of such strained and aged specimens were obtained at $90^{\circ} \mathrm{C}$ as shown in Fig. 15. ${ }^{24)}$ In the figure, a dashed curve plotted against the logarithm of aging time $t_{\mathrm{a}}$ gives the stress relaxation behavior after straining up to $\varepsilon_{\mathrm{a}}=0.04$. At the end of aging time $t_{\mathrm{a}}$, the constant rate compression of the aged specimen was resumed. In Fig. 15, the stress-strain relations of the aged specimens are drawn as the solid curves rising linearly from the dashed line and horizontally shifted by $\log t_{\mathrm{a}}$ along the axis of $\varepsilon$. A solid curve drawn from the origin of the axes $\sigma$ and $\varepsilon$ gives a plot of stressstrain relation for the annealed specimen, showing that the strain of 0.04 is a little larger than that at the limit of initial linear region of $\sigma-\varepsilon$ curve, yet significantly less than that of the yield point. As seen in Fig. 15, the yield stress of the specimens aged under the strain of 0.04 increased continuously with aging time.

The stress-strain relations of PMMA specimens annealed and aged under a compressive strain of 0.08 are shown in Fig. 16. ${ }^{24)}$ As seen from the $\sigma-\varepsilon$ relation of the annealed specimen (the solid curve drawn from the origin), the strain of 0.08 has exceeded that at the yield point. The stress relaxation in the strained state is much faster than that in the case of $\varepsilon_{\mathrm{a}}=0.04$. This faster stress relaxation indicates that the metastable structure has changed into a softened melt-like structure. Due to this softened structure, the yield stress at the resumed compression after a comparatively short time of aging was lower than that of unaged samples. However, at long times of aging the yield stress evolved to exceed that of the unaged sample.

Time evolution of yield stress similar to those shown in Figs. 15 and 16 has generally been found also in quenched amorphous polymers, and is referred to as physical aging.$^{25)}$ In Fig. $17^{24)}$ the time evolutions of yield stress $\sigma_{\mathrm{y}}$ shown in Figs. 15 and 16 are compared with that in the case of physical aging in quenched PMMA. A horizontal dash-dot line in the figure gives the magnitude of the yield stress for the annealed specimen, while a dashed line near the bottom shows the value of the yield stress of unaged quenched specimens. The mean value of yield stress obtained at a aging time of $2000 \mathrm{~h}$ (the longest aging time for quenched specimens in the experiment) is almost consistent with the stress level of the dash-dot line. This indicates that the structure in the sample quenched and aged for $2000 \mathrm{~h}$ is presumably identical with a metastable structure in annealed specimens resulted through the quite slow cooling. As shown in Fig. 17, the yield stress of annealed specimens strained by either 0.04 or 0.08 and then aged increased linearly with $\log t_{\mathrm{a}}$. In the case of $\varepsilon_{\mathrm{a}}=0.04$, the yield stress increased more than $10 \%$ of that for original annealed specimens within an aging time of $24 \mathrm{~h}$.

As seen in Fig. 17, three curves for aged specimens of the different histories seem to form a continuous master curve if they are provided with appropriate horizontal shifts. This master curve, hence, permits one to expect that the evolution of yield stress in quenched specimens at times longer than $2000 \mathrm{~h}$ possibly continues following the evolution curves for annealed and strained specimens. That is, one can suppose that the aging in quenched specimens is accelerated by the imposition of strain. This conjecture led us to the DSC examination of the aging structure in annealed and strained specimens.

In Fig. 18, ${ }^{24)}$ DSC thermograms of annealed and strained specimens are shown for the case of $\varepsilon_{\mathrm{a}}=0.08$. In this figure, endothermic peaks at temperatures below $T_{\mathrm{g}}$ are distinguishable for specimens of $t_{\mathrm{a}}=3 \mathrm{~h}$ and $24 \mathrm{~h}$. The details of these peaks are shown on an enlarged scale in Fig. 19, ${ }^{24)}$ showing that the peak evolves with increasing aging time. Another feature in Fig. 18 should be noticed: an endothermic peak appearing at a temperature above $T_{\mathrm{g}}$ is attenuated with increasing aging time as reproduced distinctly on an enlarged scale in Fig. 20. Since the endothermic peak for quenched polymer is generally known to evolve with aging time, ${ }^{24,25)}$ we

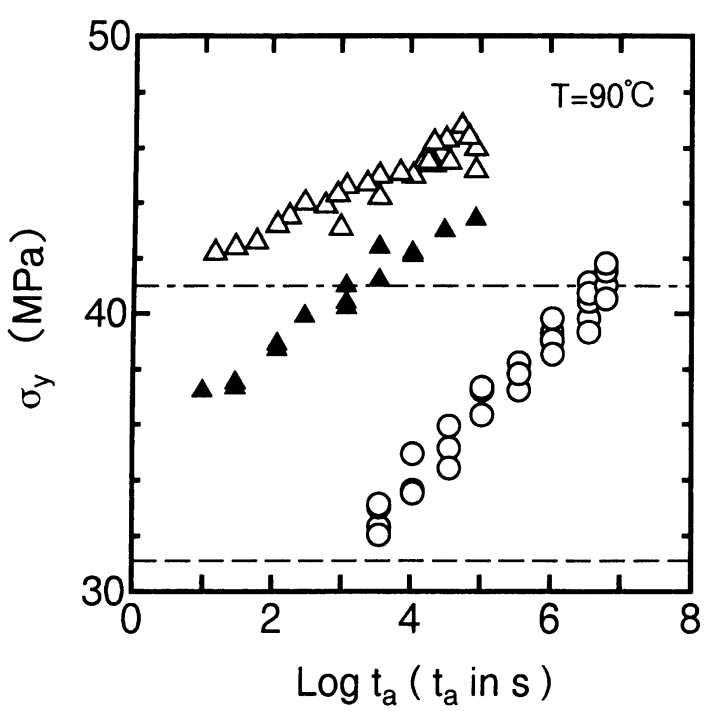

Fig. 17. Yield stress $\sigma_{\mathrm{y}}$ plotted against the logarithm of aging time $t_{\mathrm{a}}$ for three sorts of aged specimen. Symbol $\triangle$ is for annealed specimens aged under a strain of $0.04, \boldsymbol{\Delta}$ is for annealed specimens aged under a strain of 0.08 , and $\bigcirc$ is for quenched specimens aged without strain 
can conclude that the change in DSC thermograms with aging time for annealed and subsequently strained specimens is qualitatively different from that for quenched specimens. In other words, the DSC thermograms obtained for annealed and strained specimens probably indicate that the aging in annealed and strained specimens is not ascribable to the acceleration of the aging in quenched specimens, but controlled by a mechanism which may be essentially different from that of the aging in quenched amorphous polymers.

To analyze the complicated situation of the aging in the annealed and strained specimens, we measured thermally stimulated deformation recovery of the strained and then aged specimens. This is because the deformation recovery reveals qualitatively the state of strain energy stored in strained samples.

In Fig. 21, thermally stimulated deformation recovery (TSDR) for strained and aged specimens is plotted as strain recovery rate $(\mathrm{d} \varepsilon / \mathrm{d} T)$ against temperature. The specimens

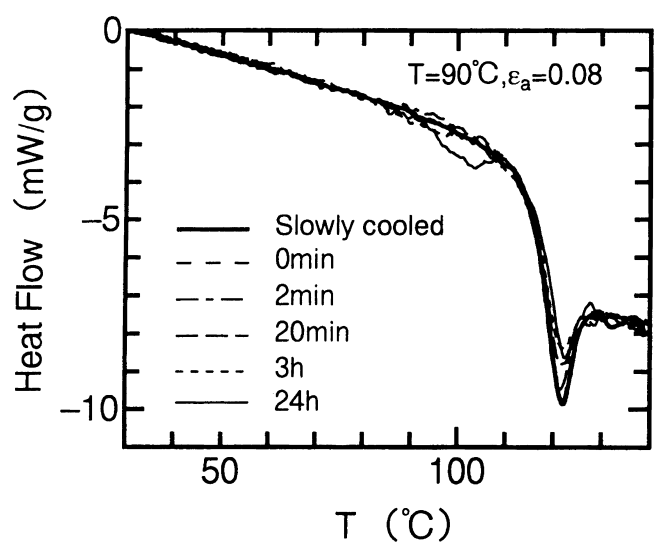

Fig. 18. Variation of DSC thermograms with aging time for PMMA annealed and aged under a strain of 0.08 .

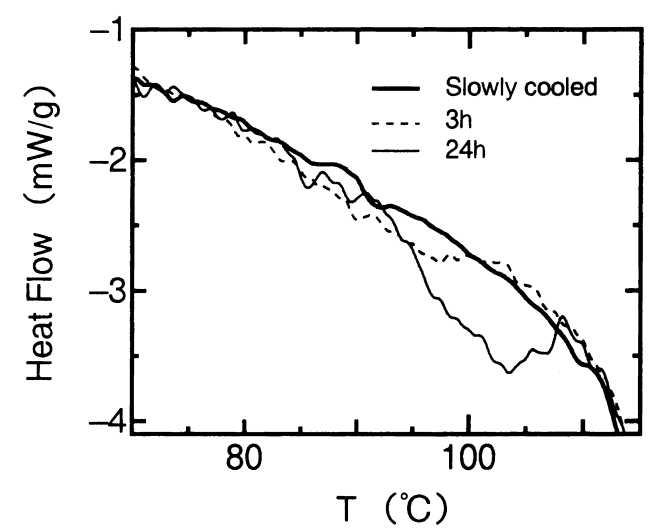

Fig. 19. Details of variation in DSC thermograms with aging time at temperatures below $T_{\mathrm{g}}$ for PMMA annealed and aged under a strain of 0.08 were strained up to 0.08 and then aged for various periods of $2 \mathrm{~min}$ to $24 \mathrm{~h}$. As seen in the figure, there appear two recovery peaks at the constant rate of heating: one is at a temperature below $T_{\mathrm{g}}$ and another is above $T_{\mathrm{g}}$. Concerning the recovery peak below $T_{\mathrm{g}}$, Oleynik ${ }^{26)}$ reported that in a temperature range around this peak some amount of strain energy stored in the sample is released. Hence, we must take into account this energy release (i.e., exothermic effect) when examining the DSC result such as shown in Fig. 19. Let us re-examine the DSC thermograms of Fig. 19 by comparing them closely with the deformation recovery shown in Fig. 21. The plot for the comparison is shown in Fig. 22. Arrow 1 on the top panel points the peak of sub- $T_{\mathrm{g}}$ deformation recovery in the case of $t_{\mathrm{a}}=3 \mathrm{~h}$. The strain energy is expected to have been released around the temperature pointed by the arrow 1 . In the same temperature range of the arrow 1 on the bottom panel, the endothermic curve has been depressed towards the exothermic direction, probably because of the thermal energy balance between the endothermic behavior caused by an aged structure brought by ordinary physical aging and the exothermic effect due to the release of strain energy. Similar depression of the endothermic behavior is seen also in the case of $t_{\mathrm{a}}=24 \mathrm{~h}$ around the temperature pointed by arrow 2 . Endothermic behavior due to the structure brought by ordinary physical aging began to appear first, and then at slightly higher temperatures (around arrow 2) the strain energy was presumably released simultaneously with the endothermic behavior. Thus, a transient part of the thermogram is first endothermic and then depressed towards the exothermic direction, indicating that the strain energy has been locked into the molecular structure

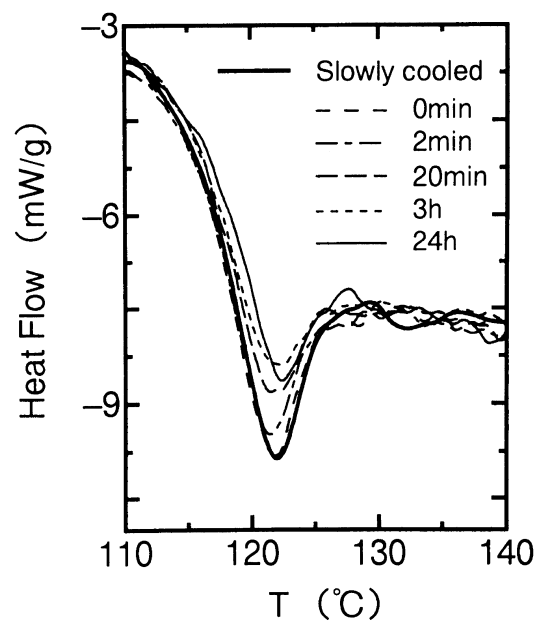

Fig. 20. Details of variation in DSC thermograms with aging time at temperatures above $T_{\mathrm{g}}$ for PMMA annealed and aged under a strain of 0.08 . 
stabilized by ordinary physical aging. That is, the strain energy could be released after the stabilized structure locking that strain energy in itself had been loosened by the thermal energy absorption.

The steady shift of the sub- $T_{\mathrm{g}}$ recovery peak to higher temperatures with aging time indicates that the strain energy comes to be locked in the stabilized structure more tightly with increasing aging time. Since the yield stress increased simultaneously with shift of the sub- $T_{\mathrm{g}}$ recovery peak to higher temperatures, we can conclude that the stabilized structure locking the strain energy in itself contributes to the significant increase in yield stress.

As shown in Fig. 20, the endothermic peak at about $122{ }^{\circ} \mathrm{C}$ was attenuated with increasing aging time. At long times, if a part of the strain energy in the stabilized structure is locked so tightly as not to be released until at a temperature above $T_{\mathrm{g}}$, then only above this temperature the locked strain energy is released. Under such condition, the energy release occurs simultaneously with endothermic behavior above $T_{\mathrm{g}}$, and thus apparently lessens the height of endothermic peak. The idea of such strain energy locked quite tightly into the aged structure allows us to mention that the endothermic peak attenuated with increasing aging time is probably results from aging in strained polymeric materials.

As seen in Fig. 21, there appeared a deformation recovery peak at about $125^{\circ} \mathrm{C}$. The peak is due to the $\alpha$ motion of polymer chains thermally stimulated at this temperature. In Fig. 20, the DSC endothermic peak appeared at about $122{ }^{\circ} \mathrm{C}$, which is a little lower than the temperature of the deformation recovery peak. This temperature difference presumably indicates such that, at temperatures above $T_{\mathrm{g}}$ the metastable structure is first loosened by thermal stimulation and nearly at

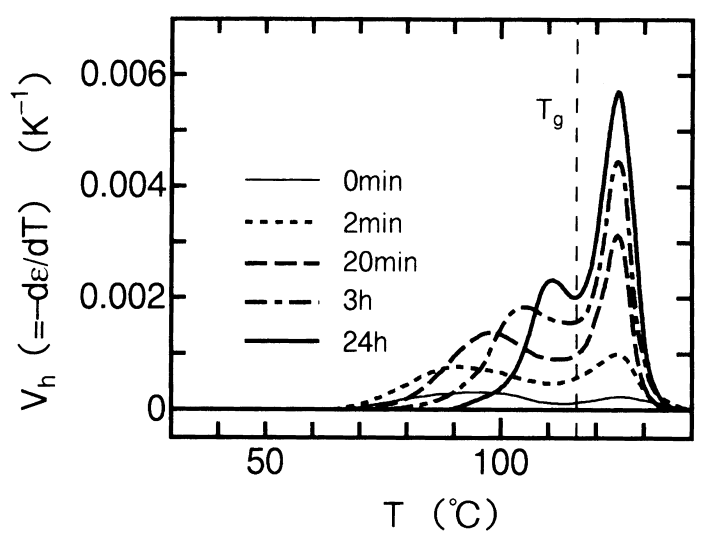

Fig. 21. Variation with aging time in plots of strain recovery rate against $T$ for PMMA annealed and aged under a strain of 0.08 . the same time the strain energy having quite tightly been locked is released. The $\alpha$ motion of polymer chains is allowed and the peak of deformation recovery is observed at the temperature a little higher than that of the endothermic peak.

\subsection{Strain Aging in Quenched PMMA and PC}

We also studied the strain aging in quenched PMMA under compressive strain ${ }^{27)}$ as well as under tensile strain ${ }^{28)}$. In these cases, experimental data obtained by DSC and TSDR indicated strain energy given to the quenched PMMA specimen to be locked into physically aged structure more tightly with aging time. In the aging under tensile strain, evolutions of tensile modulus and yield stress with aging time were much more significant than those in quenched and physically aged PMMA, giving evidence of aging process proceeding even under negative (expansive) hydrostaic pressure.

Experimental analysis on strain aging in quenched $\mathrm{PC}^{29)}$ under compressive strain showed evolution of yield stress for quenched PC aged under the strain was much faster than that for physical aging, probably due to the physically aged structure locking the strain energy tightly in itself.
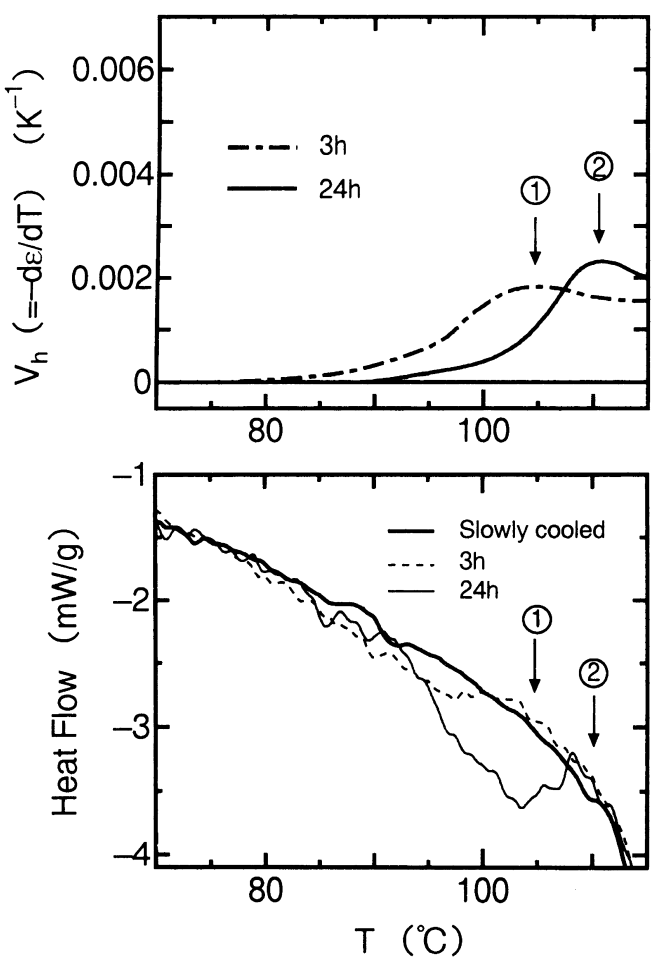

Fig. 22. Deformation recovery curves (top panel) closely compared to DSC thermograms (bottom panel) for PMMA annealed and aged under a strain of 0.08 . 


\section{APPLICABILITY OF PLASTIC DEFORMATION IN GLASSY POLYMERS TO THEIR COLD WORKING}

Glassy polymers are generally formed after heated to a temperature above the glass transition point $\left(T_{\mathrm{g}}\right)$. Yet, this heat forming technique consumes thermal energy in quantity, deteriorates the working environment due to heat, and involves complex work processes. In addition, the technique is not applicable to materials with low heat resistance, such as biodegradable polymers. To overcome these problems, the industry has been seeking a technique for forming glassy polymers at a temperature considerably lower than $T_{\mathrm{g}}$ (the technique hereinafter referred to as "cold working"). However, attempts to deform a glassy polymer largely at low temperatures result in cracking or fracture of the material, since molecular chains in the metastable material are not able to make thermal motions freely. This hampers cold working of glassy plastic materials.

As mentioned above, on the other hand, our study regarding plastic deformation in glassy polymers revealed that application of strain beyond the yield point results in nonequilibrium melt-like structure in the polymers. Such a nonequilibrium softened structure can be obtained under in uniaxial tension as well as in uniaxial compression. This implies that if a non-equilibrium structure of polymer molecules is set up in uniaxial compression, the material may subsequently be possible to deform largely in tension without causing fracture even at a low temperature.

Figure $23^{30)}$ gives nominal stress-strain curves at $30{ }^{\circ} \mathrm{C}$ for

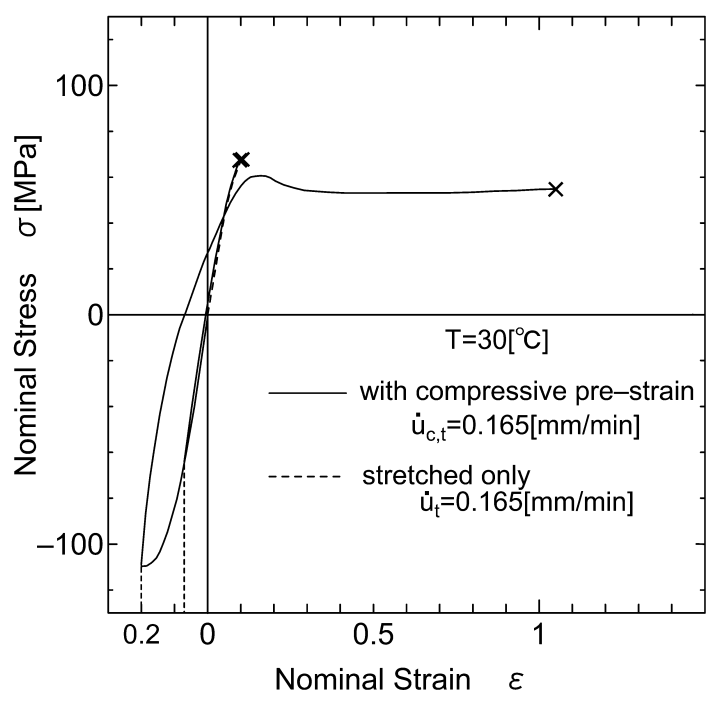

Fig. 23. Effect of pre-compression on uniaxial elongational ductility for PMMA. fully annealed PMMA samples once compressed up to a strain of 0.07 or 0.2 and subsequently stretched, compared with a stress-strain relation of the same sample in simple uniaxial tension. The cross mark $\times$ designates the fracture point of the samples. The sample stretched only exhibits a brittle fracture after the linear stress-increase, whereas the sample precompressed up to a strain of 0.2 showed a quite ductile behavior in uniaxial tension with a fracture strain more than 1.0. For the sample pre-compressed to a strain of 0.07 , on the other hand, it exhibited a brittle fracture after linear stressincrease as in the case of the sample stretched only. This is likely due to much less non-equilibrium structure induced in the material compressed to a strain of 0.07 . Accordingly, it is suggested that the non-equilibrium structure in PMMA induced by a compressive strain up to the yield point is effective for its cold working.

Time period elapsed under imposed compressive strain may influence the ductile behavior of PMMA samples in the subsequent stretching. Figure $24^{30)}$ shows change in the ductile behavior as a function of time period elapsed under a compressive strain of 0.2 . The fracture strain in subsequent tension increased with time period, indicating that some amount of time period under the compressive strain is more effective in enhancing the ductility.

The melt-like non-equilibrium structure in glassy polymers has been illustrated to be effective for their cold working. ${ }^{30)}$ Yet, the study on the application of the melt-like structure to cold working is still on the primary stage, and further intensive investigation is necessary on methods of cold working as well as on the quality of cold formed polymers.

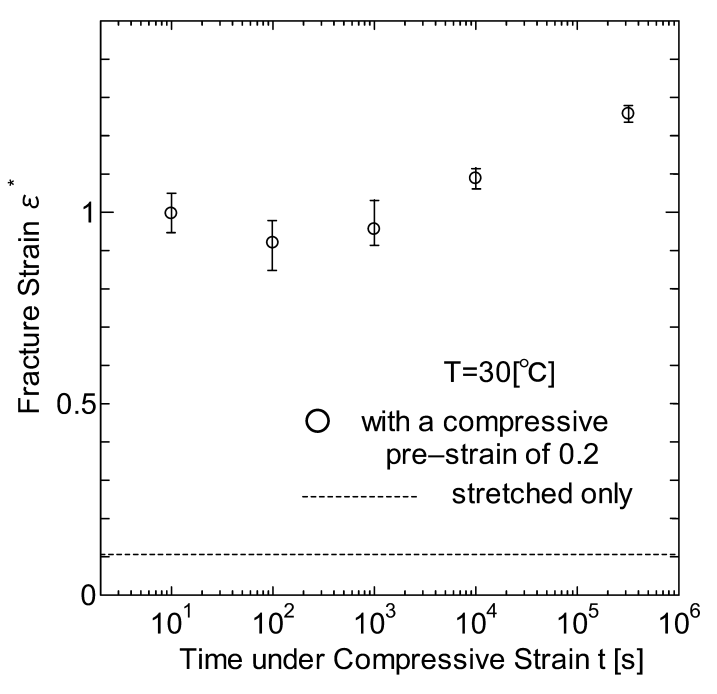

Fig. 24. Tensile fracture strain of pre-compressed samples plotted against time period elapsed under a compressive strain of 0.2 . 


\section{SUMMARY}

The steady plastic flow in the post-yield state of glassy polymers was analyzed using the Eyring equation in a novel way. This enabled us to find unique functional relations among the Eyring parameters. It was shown that the functional relation between the activation energy and activation entropy for the glass agreed well with that determined for the equilibrium melt of the polymer. This agreement convincingly gave evidence of the structural transition in the glass to the melt-like structure.

Structural relaxation due to aging in non-equilibrium meltlike structures was demonstrated using data of time-dependent mechanical property, differential scanning calorimetry and thermally stimulated deformation recovery. It was found that the strain energy given to glassy polymers during their yielding process was locked in tightly due to structural relaxation of the non-equilibrium structure.

A study on cold working of glassy polymers was presented. PMMA glass once deformed up to yield strain in uniaxial compression exhibited quite high ductility in subsequent tension probably due to non-equilibrium melt-like structure in the polymer glass. Applicability of the non-equilibrium meltlike structure in polymer glasses to their cold working was discussed.

\section{REFERENCES}

1) Roetling JA, Polymer, 6, 311 (1965); ibid, 6, 615 (1965); ibid, 7, 303 (1969).

2) Bauwens-Crowet C, Bauwens JC, Homes G, J Polym Sci, A-2, 7, 735 (1969).

3) Zitek P, Zelinger J, J Appl Polym Sci, 14, 1243 (1970).
4) Brady TE, Yeh GSY, J Macromol Sci-Phys, B9 (4), 695 (1974).

5) Hope PS, Ward IM, J Mater Sci, 16, 1511 (1981).

6) Truss RW, Clarke PL, Duckett RA, Ward IM, J Polym Sci, Polym Phys, 22, 191 (1984).

7) Robertson RE, JChem Phys, 44, 3950 (1966).

8) Rendell RW, Ngai KL, Fong GR, Yee AF, Bankert RJ, Polym Engng Sci, 27, 2 (1987).

9) Shay Jr RM, Caruthers JM, J Rheol, 30, 781 (1986).

10) Lefebvre JM, Escaig B, J Mater Sci, 20, 438 (1985).

11) Eyring H, J Chem Phys, 4, 283 (1936).

12) Glasstone $\mathrm{S}$, Laidler $\mathrm{K}$, Eyring $\mathrm{H}$, "The Theory of Rate Processes", 480-483, McGraw-Hill, NY (1941).

13) Nanzai Y, Kobunshi Ronbunshu, 41, 389 (1984).

14) Ree T, Eyring H, J Appl Phys, 26, 793 (1955).

15) Nanzai Y, Kobunshi Ronbunshu, 38, 399 (1981).

16) Nanzai Y, J Non-Crystall Sol, 131-133, 516 (1991).

17) Nanzai Y, Nihon Reoroji Gakkaishi, 10, 61 (1982).

18) Nanzai T, Polym Engng Sci, 30, 96 (1990).

19) Nanzai Y, Nihon Reoroji Gakkaishi, 20, 4 (1992).

20) Adam G, Gibbs JH, J Chem Phys, 43, 139 (1965).

21) Nanzai Y, Konishi T, Ueda S, J Mater Sci, 26, 4477 (1991).

22) Ferry JD, "Viscoelastic Properties of Polymers", 289-290, $3^{\text {rd }}$ edn, John Wiley, NY (1980).

23) Asami K, “Akuriru-jushi (Acrylic Resins)”, 84, Nikkan Kogyo, Tokyo (1970).

24) Nanzai Y, Miwa A, Cui SZ, Polym J, 32, 51 (2000).

25) Struik LCE, "Physical Aging in Amorphous Polymers and Other Materials", Elsevier, Amsterdam (1978).

26) Oleynik E, Progr Colloid Polym Sci, 80, 140 (1989).

27) Nanzai Y, Miwa A, Cui SZ, JSME Intl J, Ser A, 42, 479 (1999).

28) Cui SZ, Nanzai Y, Polym J, 33, 444 (2001).

29) Cui SZ, Nanzai Y, Yoshioka S, Kobunshi Ronbunshu, 57, 37 (2000).

30) Kinba T, Hiruma S, Nanzai Y, Zairyo, 54, 915 (2005). 